\title{
A comprehensive study of Interatomic Coulombic Decay in argon dimers: Extracting $R$-dependent absolute decay rates from the experiment
}

\author{
J. Rist ${ }^{1}{ }^{*}$ T. Miteva ${ }^{2}$, B. Gaire ${ }^{3}$, H. Sann ${ }^{1}$, F. Trinter ${ }^{1}$, M. Keiling ${ }^{1,4}$, \\ N. Gehrken ${ }^{1}$, A. Moradmand ${ }^{4}$, B. Berry ${ }^{5}$, M. Zohrabi $^{5}$, M. Kunitski ${ }^{1}$, I. \\ Ben-Itzhak $^{5}$, A. Belkacem ${ }^{3}$, T. Weber ${ }^{3}$, A. L. Landers ${ }^{4}$, M. Schöffler ${ }^{1}$, \\ J. B. Williams ${ }^{6}$, P. Kolorenč ${ }^{7}$, K. Gokhberg ${ }^{2}$, T. Jahnke ${ }^{1}{ }^{\dagger}$ and R. Dörner ${ }^{1 \ddagger}$ \\ 1 Institut für Kernphysik, Goethe Universität, \\ Max-von-Laue-Str.1, 60438 Frankfurt, Germany \\ 2 Theoretical Chemistry Group, Heidelberg University, 69117 Heidelberg, Germany \\ 3 Chemical Sciences Division, Lawrence Berkeley \\ National Laboratory, Berkeley, CA 94720, USA \\ 4 Department of Physics, Auburn University, AL 36849, USA \\ 5 J. R. Macdonald Laboratory, Department of Physics, \\ Kansas State University, Manhattan, KS 66506, USA \\ 6 Department of Physics, Reno University, NV 89557, USA \\ 7 Faculty of Mathematics and Physics, Charles University in Prague, \\ V Holešovičkách 2, 18000 Prague, Czech Republic
}

(Dated: September 8, 2016)

\begin{abstract}
In this work we present a comprehensive and detailed study of Interatomic Coulombic Decay (ICD) occurring after irradiating argon dimers with XUV-synchrotron radiation. A manifold of different decay channels is observed and the corresponding initial and final states are assigned. Additionally, the effect of nuclear dynamics on the ICD electron spectrum is examined for one specific decay channel. The internuclear distance-dependent width $\Gamma(R)$ of the decay is obtained from the measured kinetic energy release distribution of the ions employing a classical nuclear dynamics model.
\end{abstract}

\footnotetext{
* rist@atom.uni-frankfurt.de

$\dagger$ jahnke@atom.uni-frankfurt.de

‡ doerner@atom.uni-frankfurt.de
} 


\section{INTRODUCTION}

During the last 20 years Interatomic Coulombic Decay (ICD) has been established as a common decay mechanism of an electronic excitation in weakly bound matter i.e. clusters. As ICD occurs, the excitation energy of one atom or molecule of a loosely bound system is transferred to a neighboring atom or molecule of the compound. The latter energy acceptor emits an electron in order to release the excess energy. After its prediction in 1997 by the Cederbaum group [1], ICD was identified in several pioneering experiments on Ne clusters [2-4] employing different experimental techniques. Follow-up studies during the recent years showed that ICD is not an exotic process, but rather a very common decay route in nature as it occurs after excitation by photons [2-4], electrons [5], and ions [6] and in differently bound systems from van der Waals clusters, such as the extreme case of $\mathrm{He}_{2}$ [7], to systems consisting of hydrogen bonds, such as water clusters $[8,9]$. A review of an experimentalist point of view of the "early years of ICD" can be found in Ref. [10] and of more recent studies in Ref. [11]. Two comprehensive theoretical review papers by Santra et al. [12] and Averbukh et al. [13] are available, as well.

The ICD process in argon dimers or small argon clusters has been studied in the past by employing different excitation schemes prior to ICD. In pioneering work by Morishita et al. ICD in argon dimers was observed after Auger decay of a $2 p$-vacancy created by means of synchrotron radiation [14]. In follow-up work [15-17] the occurrence of ICD in triply charged breakup channels $\left(\mathrm{Ar}^{2+} / \mathrm{Ar}^{+}\right)$was examined in more detail, and in addition a radiative charge transfer was observed yielding doubly charge breakup channels [18, 19]. Similar findings were reported in electron impact studies [5, 20, 21] and studies on ICD after resonant Auger decay [22, 23].

In the present article a study of ICD at low incident photon energies is presented. Photon energies in a similar range were employed by Lablanquie et al. [24] in pioneering experiments in 2007 in order to examine the onset of different ICD channels in several rare-gas dimers. The present work was performed at a fixed energy showing the occurrence of several ICD channels after shakeup ionization. Furthermore, several decay pathways of radiative charge transfer are observed after a direct double ionization of the dimer. This is in line with aforementioned studies, where doubly ionized states were populated after Auger decay [18, 19]. 
Already very early theoretical work showed (due to the strong dependence of the decay rate on the internuclear distance $R$ of the atoms participating in ICD) a vast deviation of the ICD spectrum for different decay times [25]. Accordingly, the decay width of a IC decaying state in NeAr was determined by comparing measured ICD spectra to a set of calculations employing several different decay widths [26]. In our present work we were able to extract the $R$-dependent decay width directly from the experimental data by performing an iterative procedure using potential energy curves and the ground state vibrational wave function of the dimer as an input.

\section{EXPERIMENTAL SETUP}

The experiment was performed using a Cold Target Recoil Ion Momentum Spectroscopy (COLTRIMS) [27-29] apparatus at beamline 10.0.1 of the Advanced Light Source (ALS) in Berkeley operating in two-bunch mode. A beam of linearly polarized photons of $h \nu=51 \mathrm{eV}$ energy was crossed with a supersonic gas jet. The jet consisted of a mixture of $40 \%$ nitrogen and $60 \%$ argon expanding through a $30 \mu \mathrm{m}$ nozzle at $300 \mathrm{~K}$ at a driving pressure of 3.4 bar into vacuum. The amount of argon dimers that were generated under the given expansion conditions was of the order of a few percent. The setup consisted of a two stage supersonic gas jet yielding a target density of approximately 1e-5 mbar. The ions and electrons created in the photo reaction were measured in coincidence by guiding them onto two separate position- and time-sensitive microchannel plate detectors employing an electric field of $5.6 \mathrm{Vcm}^{-1}$. A magnetic field with a magnetic flux density of $7 \mathrm{G}$ parallel to the electric field was superimposed in order to achieve a solid angle of detection of $4 \pi$ for electrons having up to $17 \mathrm{eV}$ kinetic energy. The electron arm of the COLTRIMS analyzer consisted of a time-focusing scheme [29]. From the measured times-of-flight and positions of impact the 3-dimensional momentum vector (and accordingly the energy and angular emission direction) of all charged fragments, i.e. two electrons and two fragment ions was obtained. 


\section{RESULTS}

At a photon energy of $h \nu=51 \mathrm{eV}$ a variety of reactions may occur in an argon dimer. However, neither outer-valence ionization nor inner-valence ionization i.e. removing a $3 s$ electron, which is bound by an energy of $E=29.3 \mathrm{eV}$, can trigger ICD in $\mathrm{Ar}_{2}$, as the excitation energy of the latter ionized state is insufficient to further ionize the dimer. The energetically lowest lying states that can undergo ICD are $\mathrm{Ar}_{2}^{+}$shakeup states of the type $\mathrm{Ar}-\operatorname{Ar}^{+*}\left(3 p^{4} n l\right)$. Yet another open channel is single-site direct double ionization. The double ionization ground state $\left(\mathrm{Ar}^{2+}\left(3 p^{4}{ }^{3} P\right)-\mathrm{Ar}\right)$ is located at a threshold of $43.43 \mathrm{eV}$ and the first two single-site doubly ionized excited states $\left(\operatorname{Ar}^{2+}\left(3 p^{4}{ }^{1} D\right)-\operatorname{Ar}\right)$ and $\left(\operatorname{Ar}^{2+}\left(3 p^{4}{ }^{1} S\right)-\operatorname{Ar}\right)$ can be found at energies of $45.10 \mathrm{eV}$ and $47.48 \mathrm{eV}$ [17].

In Fig. 1 we show how our coincident detection of both electrons and both ions can be used to unambiguously disentangle these different reactions. Figure 1 a) depicts the energy of one of the measured electrons as a function of the kinetic energy release (KER) of the ions. In this type of plot horizontal lines indicate photoelectrons from the population of states which can decay by ICD as their energy is independent of the KER. The first excitation levels of the singly charged $3 p$-ionized Ar atom are in the range of $19.7 \mathrm{eV}$ to $25.4 \mathrm{eV}$. Accordingly, photoelectron features can be expected in an energy range of $9.8 \mathrm{eV}$ to $15.5 \mathrm{eV}$. However no horizontal photoelectron line is apparent in Fig. 1 a). Instead only a single broad feature is visible, which is due to the limited electron energy resolution of our experimental setup. On the other hand a number of diagonal lines can be found, belonging to a constant sum of the electron kinetic energy and the KER. These lines occur as the excitation energy of the decaying $\mathrm{Ar}_{2}^{+*}$ is shared between the kinetic energy of the emitted electron and the two ions, which is the case, for example, in ICD.

In Fig. 1 b) the sum kinetic energy of the two electrons is depicted versus the KER. In this representation ICD can be distinguished from processes in which an additional photon is emitted. Events of ICD appear in the upper part of the figure between $12 \mathrm{eV}$ and $18 \mathrm{eV}$ as diagonal lines. For these events the sum energy of all fragments is a constant. The two horizontal lines located at an electron sum energy of $6 \mathrm{eV}$ and $8 \mathrm{eV}$ on the contrary show no dependence on the KER. A constant sum kinetic energy of the two electrons is an evidence for double ionization of one atom of the dimer followed by an electron transfer from the 
a)

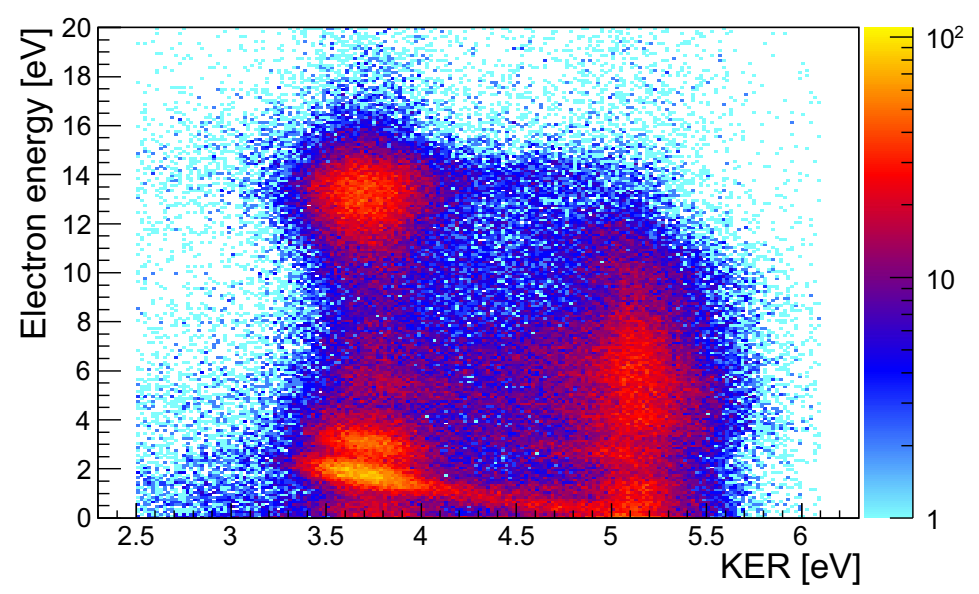

b)

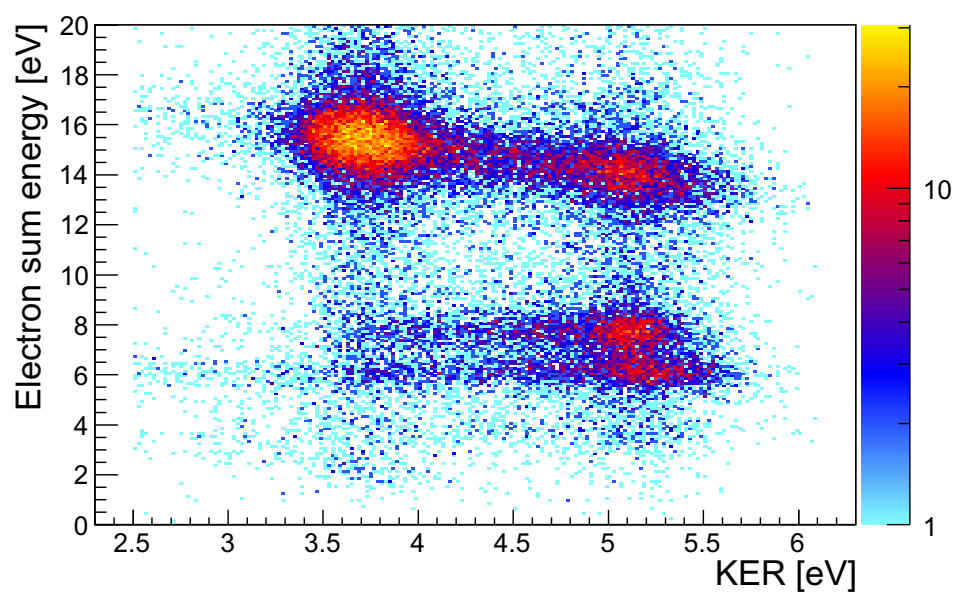

c)

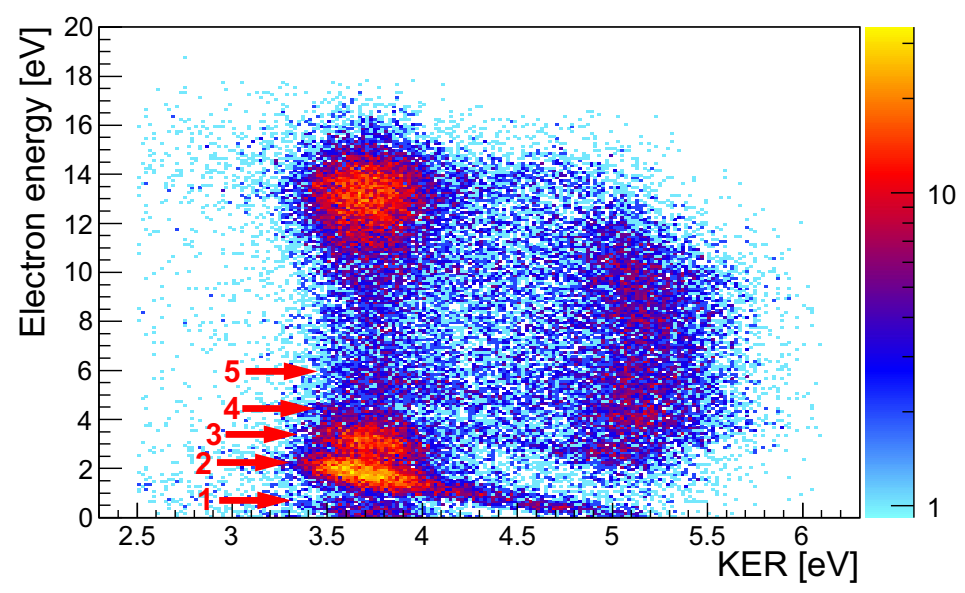

FIG. 1. (Color online) a): Experimental yield as function of the electron energy and the kinetic energy release plotted on a logarithmic scale; b): Yield as function of the sum kinetic energy of the two electrons and the kinetic energy release. The diagonal lines between $12 \mathrm{eV}$ and $18 \mathrm{eV}$ occur due to ICD, while the horizontal lines between $4 \mathrm{eV}$ and $10 \mathrm{eV}$ belong to direct double ionization; c): Yield as in a) with an additional restriction that the sum energy of the two electrons is between $12 \mathrm{eV}$ and $18 \mathrm{eV}$ and therefore just events from the upper diagonal ICD line in b) are plotted. Red arrows indicate the position of five separate lines corresponding to different sum energies of electron energy and kinetic energy release. 
other (neutral) atom and the simultaneous emission of a photon balancing the energy and a Coulomb explosion of the remaining doubly charged $\mathrm{Ar}_{2}^{2+}$ dimer. According to their energy values, the two horizontal lines belong to the double ionization into the $\mathrm{Ar}_{2}^{2+}$ ground state and the first excited $\mathrm{Ar}_{2}^{2+*}$ state.

Figure 1 c) shows a subset of the data presented in Fig. 1 a) with a gate on the the diagonal ICD line in Fig. 1 b). Compared to Fig. 1 a) the relative intensity in the region of high KER values of approximately $5.2 \mathrm{eV}$ is significantly reduced. Using the reflection approximation $[30,31]$ the internuclear distance $R$ of the two atoms at the instant of the decay can be obtained from the KER (in atomic units) by $R=1 / \mathrm{KER}$. A value of $3.7 \mathrm{eV}$ corresponds to an internuclear distance of $3.9 \AA$ (7.4 a.u.), which is very close to the mean internuclear distance of the ground state of the argon dimer [32]. As most of the dimer shakeup states have a slightly attractive potential in the Franck-Condon region the dimer contracts during ICD. The main contribution to the decay seems to occur already at larger distances. The increased intensity at a KER of approximately $5.2 \mathrm{eV}$ corresponds to a smaller internuclear distance of $2.8 \AA$ (5.2 a.u.) indicating the inner turning point of the excited $\mathrm{Ar}^{+*}-\mathrm{Ar}$ potential energy curve (PEC). A total of five diagonal lines are visible in Fig. $1 \mathrm{c}$ ) having sum energies of approximately (1) $4.0 \mathrm{eV}$, (2) $5.2 \mathrm{eV}$, (3) $6.9 \mathrm{eV}$, (4) $7.5 \mathrm{eV}$, and (5) $9.2 \mathrm{eV}$, respectively. Line (2) appears to consist of an overlap of a shorter line similar to line (3) and a longer line similar to (4) and (5). Comparing the measured energy values to the PECs shown in Fig. 2 a) yielded the assignment of the decaying states listed in Table I. The assignments are in line with those done in [33].

The potential curves of the $\operatorname{Ar}^{+*}\left(3 p^{-2} 4 d\right)$-Ar states were approximated by averages of the PECs of the parent $\operatorname{Ar}^{2+}\left(3 p^{-2}\right)$-Ar states (see Ref. [34]). The potential energy curves of the remaining ionization satellites and the final two-site dicationic states of $\mathrm{Ar}_{2}$ were computed using the configuration interaction singles doubles (CISD) method as implemented in the GAMESS-US package [35, 36]. We employed the cc-pVDZ [37] basis set augmented with four diffuse $s$ and $d$, and two compact $d$ functions [38]. In the case of the $\operatorname{Ar}^{+*}\left(3 p^{-2} 4 p\right)-\operatorname{Ar}$ satellites, we augmented the basis set further, adding two diffuse $p$ functions.

For a better comparison of PECs and measured data, the electron energy is plotted versus the internuclear distance of the atoms in Fig. 2 b). The blue dashed line in Fig. 2 c) shows the $\mathrm{Ar}_{2}$ ground state wave function. The comparison with the measured distribution highlights that for all ICD channels the dimer contracts. This is in line with the PECs 


\begin{tabular}{|c|c|c|c|}
\hline Line & Measured sum energy $[\mathrm{eV}]$ & State & Calculated sum energy $[\mathrm{eV}]$ \\
\hline$(1)$ & 4.0 & $\left(3 p^{-2}{ }^{3} P\right) 4 p^{\mathbf{2}} \boldsymbol{D}$ & 4.06 \\
$(2.1)$ long & 5.2 & $\left(3 p^{-2}{ }^{1} D\right) 4 p^{\mathbf{2}} \boldsymbol{F}$ & 5.40 \\
$(2.2)$ short & 5.5 & $\left(3 p^{-2}{ }^{1} D\right) 3 d^{\mathbf{2}} \boldsymbol{D}$ & 5.63 \\
$(3)$ & 6.9 & $\left(3 p^{-2}{ }^{1} D\right) 3 d^{2} \boldsymbol{S}$ & 7.07 \\
$(4)$ & 7.5 & $\left(3 p^{-2}{ }^{3} P\right) 4 d^{\mathbf{2}} \boldsymbol{P}$ & 7.84 \\
$(5)$ & 9.2 & $\left(3 p^{-2}{ }^{1} D\right) 4 d^{\mathbf{2}} \boldsymbol{S}$ & 9.69 \\
\hline
\end{tabular}

TABLE I. Assignment of possible states belonging to the diagonal lines in Fig. $1 \mathrm{c}$ ).

shown in Fig. 2 a) which are all attractive in the Franck-Condon region.

\section{EXTRACTING THE ABSOLUTE DECAY WIDTH}

For theoretical considerations, the decay width as function of $R$ and the potential energy surfaces are the primary building blocks for the prediction of experimental observables like the distribution of KERs and electron kinetic energies. The interplay of nuclear dynamics and the $R$-dependent decay width leads to the non-exponential decay of the metastable state and makes the KER and electron energy spectra often very sensitive probes of the absolute value of the decay width. Prominent examples can be found in [25, 39, 40]. Examining NeAr dimers O'Keeffe et. al [41] obtained indirect evidence for the ICD lifetime from the broadening of the electron kinetic energy distribution towards lower energies and Ouchi et al. [26] used even the relative height of two peaks in the KER distribution to verify the calculated decay width directly. Here we invert this procedure and extract absolute decay widths from our data using calculated PECs.

For our procedure, an energetically isolated state is needed for which the PEC is known and the KER distribution must be experimentally separated from that of other states. The $\left({ }^{1} D\right) 4 d^{2} S$ intermediate state satisfies this requirement. We filter events corresponding to the $\left({ }^{1} D\right) 4 d^{2} S$ intermediate state (i.e. line (5) in Fig. $\left.1 \mathrm{c}\right)$ ) by requiring a sum kinetic energy of $8.3 \mathrm{eV}<E_{I C D}+K E R<9.8 \mathrm{eV}$. For these events we calculate the internuclear distance using the reflection approximation (Fig. 2 c)).

For our inversion procedure we create a one dimensional histogram of internuclear distances $R$ consisting of $K$ discrete intervals $\Delta R$. K corresponds to the number of bins in 


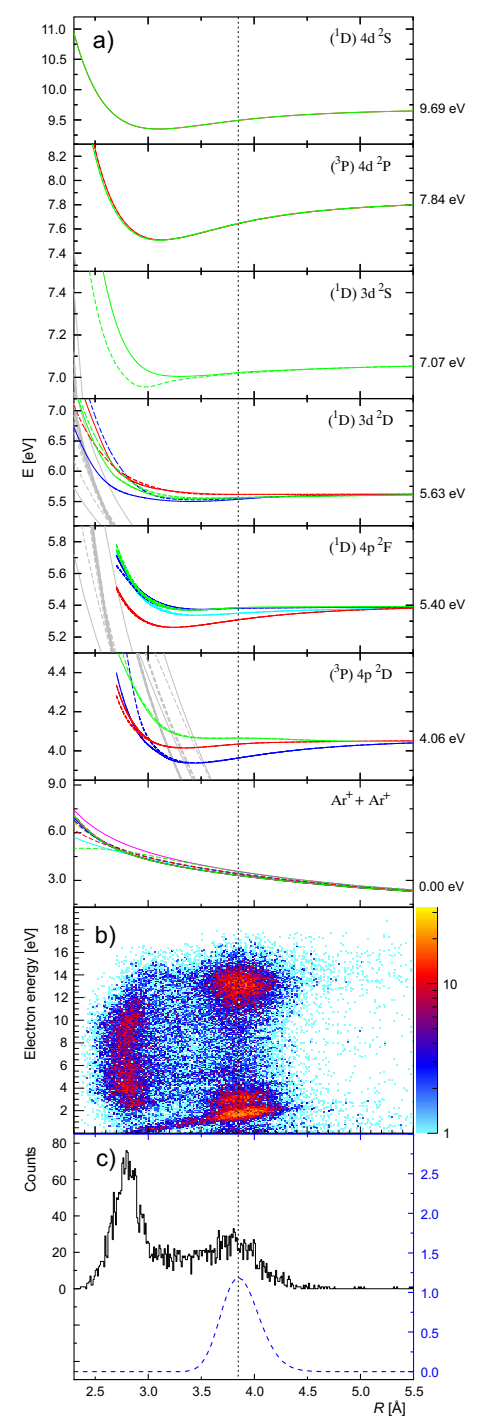

FIG. 2. (Color online) a): Potential energy curves for selected intermediate states of the argon dimer of the type $\mathrm{Ar}-\mathrm{Ar}^{+}\left(3 p^{4} X\right)$ (upper six panels). The respective states $\mathrm{X}$ are indicated in the upper right corner of each panel. The energy axis is shifted to the $\operatorname{Ar}^{+}\left(3 p^{5}\right)-\operatorname{Ar}^{+}\left(3 p^{5}\right)$ (i.e. the lowest panel) threshold so that it indicates the states' excess energy which is then converted into ICD electron energy and KER. The dissociating $\mathrm{Ar}^{+}\left(3 p^{5}\right)-\mathrm{Ar}^{+}\left(3 p^{5}\right)$ states are depicted in the upper panels in gray; b): Electron energy versus internuclear distance obtained within the reflection approximation (see text); c): The upper curve (solid black) depicts the internuclear distance distribution of events decayed from the $\left({ }^{1} D\right) 4 d^{2} S$ state. Below that the calculated $\mathrm{Ar}_{2}$ dimer ground state wave function density is indicated (dashed blue).

the histogram in Fig. 2 c). In the following we denote the $i$ th bin of the histogram as $\operatorname{Bin}_{i}$ 
(where $i=1$ corresponds to the largest value of $R$ and larger $i$ belong to smaller $R$ ). The histogram is initialized with $N$ test particles with a reduced mass $\mu$ where the total amount of test particles $(N=4442)$ is determined by the total number of experimentally observed decays (i.e. the sum of all entries in the histogram shown in Fig. 2 c), top). The $N$ particles are distributed in the histogram according to the shape of the vibrational wave function of the Argon dimer ground state (blue dashed line in Fig. 2 c)). The number of particles initially occurring in $\operatorname{Bin}_{i}$ is denoted as $n_{i}$, so that $N=\sum_{i=0}^{K} n_{i}$.

Because the PEC of the studied decay channel is solely attractive within the FrankCondon region and all particles are initialized without any initial momentum, all test particles will move towards smaller distances on propagation. The particles from $\left.\operatorname{Bin}_{i}\right|_{i=1}$ (i.e. largest $R$ ) are now propagated along the $\left({ }^{1} D\right) 4 d{ }^{2} S$ potential energy curve using classical mechanics. The time that is needed to reach the internuclear distance of the next bin, i.e. $\left.B i n_{i}\right|_{i=2}$, is labeled as $\left.t_{i}\right|_{i=1}$. In order for the extraction method to work, a further assumption has to be made: the majority of the vibrational wave function density has to decay within the first vibrational cycle of the nuclear motion and accordingly none of the test particles move towards larger $R$. With this approach the decay width $\Gamma$ can now be calculated at the distance $R_{1}$ corresponding to the $\left.\operatorname{Bin}_{i}\right|_{i=1}$ by dividing the number of measured decays $D_{i}$ in $\left.B i n_{i}\right|_{i=1}$ by the number of test particles $\left.n_{i}\right|_{i=1}$ in that bin and their residence time $\left.t_{i}\right|_{i=1}$.

$$
\Gamma^{i}=\left.\frac{D_{i}}{n_{i} \cdot t_{i}}\right|_{i=1}
$$

The test particles that did not decay in bin $i$ are fed into the next bin $i+1$. However, as the particles initialized in bin $i+1$ have zero velocity and the particles originating from bin $i$ have a velocity corresponding to the difference in the potential energy between $R_{i}$ and $R_{i+1}$, they need to be treated differently during the further steps of the extraction algorithm. Therefore, two further variables $\tilde{n}_{i}^{j}$ and $\tilde{t}_{i}^{j}$ are needed: $\tilde{n}_{i}^{j}$ denotes the number of particles present in bin $j$ which were originally initialized in bin $i . \tilde{t}_{i}^{j}$ denotes the time that particles originally initiated in bin $i$ need to move from bin $j$ to bin $j+1$. With these definitions, the number of particles fed into the next bin is given as: $\tilde{n}_{i}^{i+1}=n_{i}-D_{i}=\left.n_{i} \cdot\left(1-\Gamma^{i} \cdot t_{i}\right)\right|_{i=1}$.

The general equations for processing the following bins are: 


$$
\tilde{n}_{i}^{j}=n_{i} \cdot \prod_{l=1}^{j-1}\left(1-\Gamma^{l} \cdot \tilde{t}_{i}^{l}\right)
$$

Accordingly, the number of particles which were originally initialized in bin $i$ and are now present in bin $j$ is equal to the number of particles initiated in bin $i$ minus all particles that decayed between bin $i$ and bin $j$. Furthermore:

$$
\Gamma^{j}=\frac{D_{j}}{\sum_{i=1}^{j} \tilde{n}_{i}^{j} \cdot \tilde{t}_{i}^{j}}
$$

The decay width belonging to bin $j$ is therefore the number of measured decays in bin $j$ divided by the number of particles present in bin $j$ (which includes particles from all previous bins) and their respective residence times. Note that for the calculation of $\Gamma^{j}$ all values of $\tilde{n}_{i}^{j}$ are needed while for the calculation of $\tilde{n}_{i}^{j}$ the values of $\Gamma^{j-1}$ are sufficient.

Performing this procedure results in the experimentally determined decay rates shown in Fig. 5 together with the theoretical prediction. The latter theoretical calculation was performed using the Fano-ADC-Stieltjes method [42]. The bound and continuum parts of the resonance states as well as the coupling between them were constructed using the extended $\mathrm{ADC}(2) \mathrm{x}$ scheme for the one-particle propagator [43]. The basis set on each atom consisted of an effective core potential (ECP) with $4 s, 4 p, 4 d$, and $1 f$ basis functions with 8 active valence electrons [44]. The basis was further augmented by 15s, 15p, 13d, $7 f$, and $5 g$ diffuse functions on the atomic centers and additional sets of $5 s, 5 p$, and $5 d$ functions on 5 ghost atoms on the interatomic axis. The diffuse functions were specifically designed for the computation of Rydberg and continuum states [45].

As Fig. 5 shows, the agreement between the $a b$ initio calculation and the $\Gamma_{R}$ values extracted from the data is rather poor. This is partly in line with the findings in Ref. [26], where the ab initio treatment overestimated the decay width, as well. However, in the present case not only do the overall values differ by a factor of 10 and more, but the qualitative slope of the $R$-dependence is also different. As noted before some strong simplifications as neglecting the initial momentum distribution, all quantum mechanical coherence effects and assuming the decay will take place within the first vibrational cycle of the vibrational wave packet were made which could easily cause such disagreement between experiment and theory. In order to check the validity of our classical extraction method for obtaining the $R$-dependent decay width from the measured KER distribution, we computed the KER 
spectra produced in the decay of the $\operatorname{Ar}^{+}\left(3 p^{-2}\left({ }^{1} D\right) 4 d^{2} S\right) \operatorname{Ar}$ state quantum mechanically using different decay widths: the one extracted from the experimental data, the ab initio decay widths corresponding to the $\Sigma_{g}^{+}$and $\Sigma_{u}^{+}$states (see Fig. 5) and the ab initio decay widths scaled such that they equal the experimental one at the equilibrium distance of $\operatorname{Ar}_{2}$, $R_{e q}=3.8 \AA$. For all calculations we used the $a b$ initio PECs of the ground, decaying and final states shown in Fig. 2 a).

The partial KER spectra corresponding to a particular decay channel

$$
\sigma_{i}^{k}\left(E_{K E R}\right)=2 \pi \int_{0}^{\mathrm{inf}} d t^{\prime}\left|\left\langle E_{f_{k}}\left|\hat{W}_{d_{i} \rightarrow f_{k}}\right| \psi_{d_{i}}\left(t^{\prime}\right)\right\rangle\right|^{2}
$$

were computed following the procedure described in Ref. [46]. Here $\left|E_{f_{k}}\right\rangle$ are the eigenfunctions of the Hamiltonian of nuclear motion in the final state $k, \hat{H}_{f_{k}}$. The operator $\hat{W}_{d_{i} \rightarrow f_{k}}$ is related to the partial decay width of the state $i$ to the final state $k$ as $\Gamma_{d_{i} \rightarrow f_{k}}(R)=2 \pi\left|W_{d_{i} \rightarrow f_{k}}(R)\right|^{2}$ within the local approximation [47]. The time evolution of the nuclear wave packet of the decaying state $\left|\psi_{d_{i}}(t)\right\rangle$ is obtained by solving the time-dependent Schrödinger equation

$$
i\left|\dot{\psi_{d_{i}}}(t)\right\rangle=\left(\hat{H}_{d_{i}}-i \frac{\hat{\Gamma}_{d_{i}}}{2}\right)\left|\psi_{d_{i}}(t)\right\rangle
$$

where $H_{d_{i}}$ is the Hamiltonian of the decaying state and $\Gamma_{d_{i}}$ denotes the total decay width of the decaying state $i$. The total KER spectra corresponding to a particular decaying state were obtained as a sum of the partial KER spectra corresponding to all possible decay channels (see Ref. [34])

$$
\sigma_{i}\left(E_{K E R}\right)=\sum_{k=1}^{N_{c}} \sigma_{i}^{k}\left(E_{K E R}\right)
$$

Subsequently, we calculated the distance distributions from the total KER spectra using the reflection approximation $R=1 / K E R$.

In Figs. 3 and 4 we show the experimental and computed KER and distance distributions for the the $\mathrm{Ar}^{+}\left(3 p^{-2}\left({ }^{1} D\right) 4 d^{2} S\right) \mathrm{Ar}$ state. There is a satisfactory comparison between the experimental KER spectrum and the one computed using the experimental decay width which confirms the validity of our classical model for the extraction of the $R$-dependent decay width. Both spectra have a double-peak structure reflecting the interplay between ICD and nuclear dynamics. On the contrary, the KER spectrum computed using the theoretical decay width exhibits a pronounced peak around $3.8 \mathrm{eV}$, indicating that due to the larger value of the computed decay width, the decay occurs mainly around the equilibrium distance with 
little dynamics taking place. Uniformly scaling the ab initio decay widths corresponding to the $\Sigma_{g}^{+}$and $\Sigma_{u}^{+}$states by a factor of 0.228 and 0.271 , respectively, results in an enhancement of the second peak at higher energies. However, the ratio between the two peaks differs from that of the ones observed in the experimental spectra because the experimental decay width increases faster at shorter distances compared to the theoretical one (see Fig. 5).

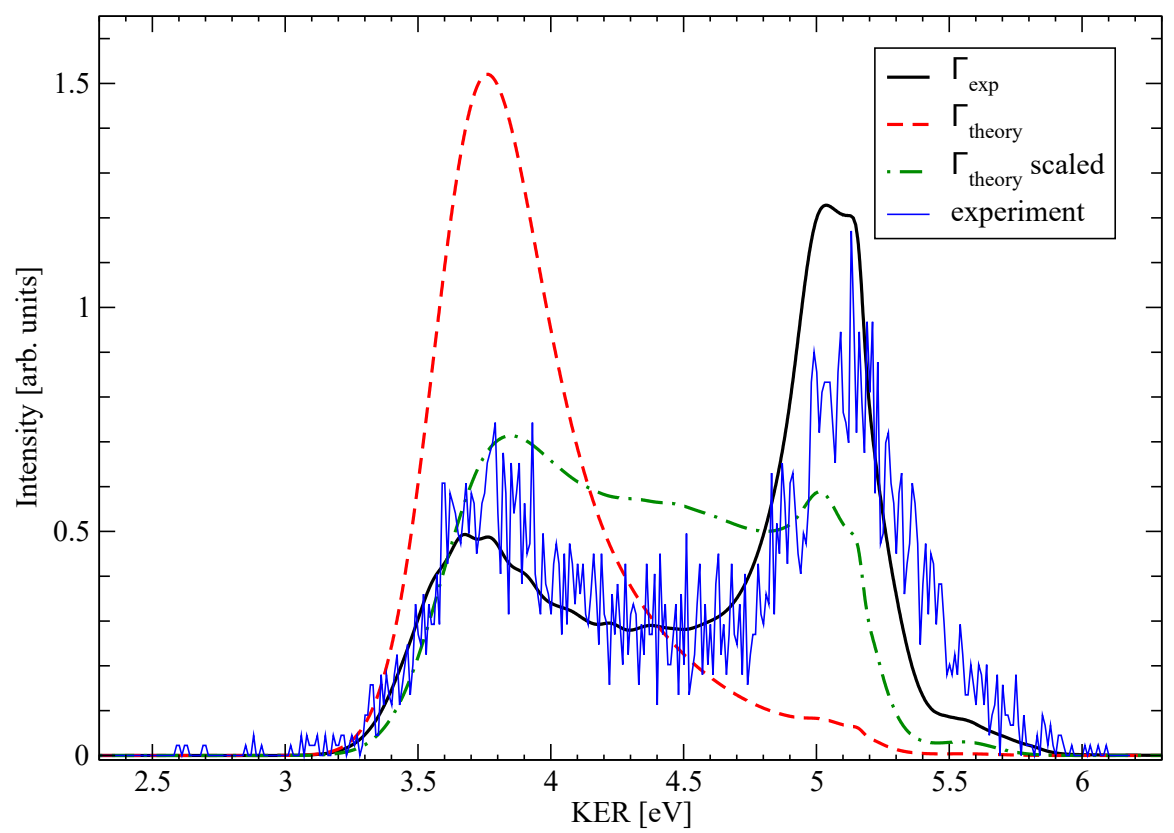

FIG. 3. Comparison between experimental and computed KER spectra produced in the decay of the $\operatorname{Ar}^{+}\left(3 p^{-2}\left({ }^{1} S\right) 4 d^{2} D\right)$ Ar state. The theoretical KER spectra were computed using the experimental decay width (black solid line) theoretical decay widths (red dashed line), and the theoretical decay widths scaled such that they equal the experimental one at the equilibrium interatomic distance $R_{e q}=3.8 \AA$ (green dashed-dotted line). All spectra were scaled such that their area is equal to 1.0

At distances around and greater than $4 \AA$ increasing decay rates can be seen in the experimentally derived values, which is contrary to the prediction and physical intuition. We believe those increasing values to be a result of underestimating the initial particle numbers $n_{i}$ at the falling edge of the ground state wave function density as well as the residence times which are calculated for zero momentum particles instead of particles with a momentum distribution. However, already around the center of the Franck-Condon region at $3.85 \AA$ our inverting procedure is rather insensitive to the details of the PEC and to 


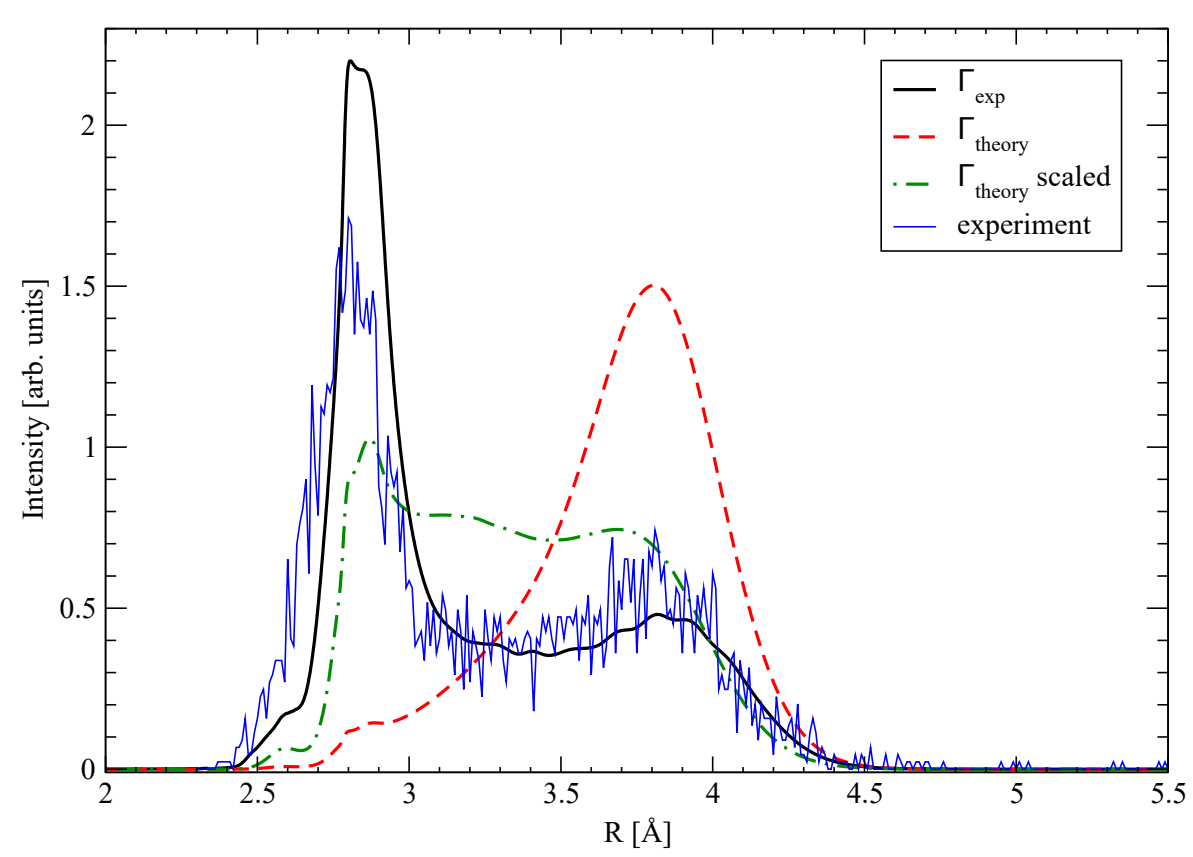

FIG. 4. Comparison between experimental and computed distance distributions produced in the decay of the $\operatorname{Ar}^{+}\left(3 p^{-2}\left({ }^{1} S\right) 4 d^{2} D\right)$ Ar state. The theoretical distance distributions were computed using the experimental decay width (black solid line) theoretical decay widths (red dashed line), and the theoretical decay widths scaled such that they equal the experimental one at the equilibrium interatomic distance $R_{e q}=3.8 \AA$ (green dashed-dotted line). All spectra were scaled such that their area is equal to 1.0 .

small variations in position and width of the initial distribution of test particles. The most likely reason for the discrepancy between the experiment and the theory is a failure of the $\mathrm{ADC}(2) \mathrm{x}$ scheme to reproduce with sufficient accuracy the wave function of the intermediate $\left(3 p^{-2}{ }^{1} D\right) 4 d^{2} S$ ionization satellite state, in particular the spatial extent of the $4 d$ orbital. On the experimental side, the use of a purely classical nuclear dynamics model in the inversion procedure can also result in some inaccuracy. However, the fall of the experimental rates in the region below $3.7 \AA$ is sound and our experiment confirms directly the strong $R$ dependence of ICD so far only known from theory.

In conclusion, we have given a comprehensive overview of photon-induced double ionization of Ar dimers at low photon energies. The coincident detection of both electrons and ions allowed to separate radiative charge transfer following single-site double ionization from the contribution of ICD following shakeup single ionization. From the measured KER distribu- 


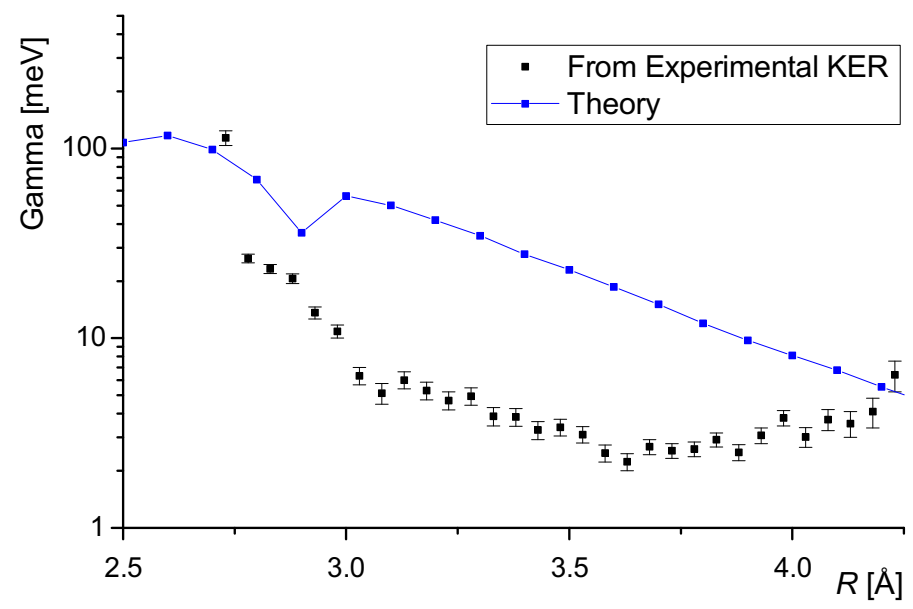

FIG. 5. (Color online) $\Gamma$ extracted from KER (black squares) compared to theoretical ab initio calculations (blue line). Statistical errors in $\Gamma$ are indicated.

tion we obtained absolute decay rates for the ICD as a function of the internuclear distance. These experimental decay rates show the expected strong dependence on the internuclear distance but are significantly smaller than predicted by the theoretical model.

\section{ACKNOWLEDGMENTS}

We thank the staff of the Advanced Light Source, in particular beamline 10.0.1 scientists for their outstanding support. This research used the Advance Light Source and resources of the National Energy Research Scientific Computing Center, DOE Offices of Science User Facilities supported by the Director, Office of Science, Office of Basic Energy Sciences, the Division of Chemical Sciences, Geosciences, and Biosciences of the U.S. Department of Energy at LBNL under Contract No. DE-AC02-05CH11231. JRML personnel were supported by Grant No.DE-FG02-86ER13491 from the same funding agency. The Auburn University personnel were supported by the National Science Foundation under contract NSF-PHYS1404366. We acknowledge the financial support of the DAAD and DFG, this 
work was performed within the DFG research unit FOR1789.

[1] L. S. Cederbaum, J. Zobeley, and F. Tarantelli. Giant Intermolecular Decay and Fragmentation of Clusters. Phys. Rev. Lett., 79(24):4778-4781, 1997.

[2] S. Marburger, O. Kugeler, U. Hergenhahn, and T. Möller. Experimental Evidence for Interatomic Coulombic Decay in Ne Clusters. Phys. Rev. Lett., 90(20), 2003.

[3] T. Jahnke, A. Czasch, M. S. Schöffler, S. Schössler, A. Knapp, M. Käsz, J. Titze, C. Wimmer, K. Kreidi, R. E. Grisenti, A. Staudte, O. Jagutzki, U. Hergenhahn, H. Schmidt-Böcking, and R. Dörner. Experimental Observation of Interatomic Coulombic Decay in Neon Dimers. Phys. Rev. Lett., 93(16), 2004.

[4] G. Öhrwall, M. Tchaplyguine, M. Lundwall, R. Feifel, H. Bergersen, T. Rander, A. Lindblad, J. Schulz, S. Peredkov, S. Barth, S. Marburger, U. Hergenhahn, S. Svensson, and O. Björneholm. Femtosecond Interatomic Coulombic Decay in Free Neon Clusters: Large Lifetime Differences between Surface and Bulk. Phys. Rev. Lett., 93:173401, 2004.

[5] X. Ren, E. Jabbour Al Maalouf, A. Dorn, and S. Denifl. Direct evidence of two interatomic relaxation mechanisms in argon dimers ionized by electron impact. Nat. Commun., 7:11093, 2016.

[6] J. Titze, M. S. Schöffler, H.-K. Kim, F. Trinter, M. Waitz, J. Voigtsberger, N. Neumann, B. Ulrich, K. Kreidi, R. Wallauer, M. Odenweller, T. Havermeier, S. Schössler, M. Meckel, L. Foucar, T. Jahnke, A. Czasch, L. Ph H. Schmidt, O. Jagutzki, R. E. Grisenti, H. SchmidtBöcking, H. J. Lüdde, and R. Dörner. Ionization Dynamics of Helium Dimers in Fast Collisions with $\mathrm{He}^{++}$. Phys. Rev. Lett., 106(3):033201, 2011.

[7] T. Havermeier, T. Jahnke, K. Kreidi, R. Wallauer, S. Voss, M. Schöffler, S. Schössler, L. Foucar, N. Neumann, J. Titze, H. Sann, M. Kühnel, J. Voigtsberger, J. H. Morilla, W. Schöllkopf, H. Schmidt-Böcking, R. E. Grisenti, and R. Dörner. Interatomic Coulombic Decay following Photoionization of the Helium Dimer: Observation of Vibrational Structure. Phys. Rev. Lett., 104(13):133401, 2010.

[8] T. Jahnke, H. Sann, T. Havermeier, K. Kreidi, C. Stuck, M. Meckel, M. Schöffler, N. Neumann, R. Wallauer, S. Voss, A. Czasch, O. Jagutzki, A. Malakzadeh, F. Afaneh, Th. Weber, H. Schmidt-Böcking, and R. Dörner. Ultrafast energy transfer between water molecules. Nat. 
Phys., 6(2):139-142, 2010.

[9] M. Mucke, M. Braune, S. Barth, M. Förstel, T. Lischke, V. Ulrich, T. Arion, U. Becker, A. Bradshaw, and U. Hergenhahn. A hitherto unrecognized source of low-energy electrons in water. Nat. Phys., 6(2):143-146, 2010.

[10] U. Hergenhahn. Interatomic and intermolecular coulombic decay: The early years. J. Electron. Spectrosc. Relat. Phenom., 184:78, 2011.

[11] T. Jahnke. Interatomic and intermolecular Coulombic decay: the coming of age story. $J$. Phys. B: At. Mol. Opt. Phys., 48:082001, 2015.

[12] R. Santra and L. S. Cederbaum. Non-Hermitian electronic theory and applications to clusters. Phys. Rep., 368:1, 2002.

[13] V. Averbukh, Ph. V. Demekhin, P. Kolorenč, S. Scheit, S. D. Stoychev, A. I. Kuleff, Y.-C. Chiang, K. Gokhberg, S. Kopelke, N. Sisourat, and L. S. Cederbaum. Interatomic electronic decay processes in singly and multiply ionized clusters. J. Electron. Spectrosc. Relat. Phenom., 183:36, 2011.

[14] Y. Morishita, X.-J. Liu, N. Saito, T. Lischke, M. Kato, G. Prümper, M. Oura, H. Yamaoka, Y. Tamenori, I. H. Suzuki, and K. Ueda. Experimental Evidence of Interatomic Coulombic Decay from the Auger Final States in Argon Dimers. Phys. Rev. Lett., 96(24), 2006.

[15] K. Ueda, H. Fukuzawa, X.-J. Liu, K. Sakai, G. Prümper, Y. Morishita, N. Saito, I. H. Suzuki, K. Nagaya, H. Iwayama, M. Yao, K. Kreidi, M. Schöffler, T. Jahnke, S. Schössler, R. Dörner, Th. Weber, J. Harries, and Y. Tamenori. Interatomic Coulombic decay following the Auger decay: Experimental evidence in rare-gas dimers. J. Electron. Spectrosc. Relat. Phenom., 166-167:3-10, 2008.

[16] K. Ueda. Photoemission and coincidence studies on gas-phase molecules. Appl. Phys. A, 92:487, 2008.

[17] S. D. Stoychev, A. I. Kuleff, F. Tarantelli, and L. S. Cederbaum. On the doubly ionized states of $\mathrm{Ar}_{2}$ and their intra- and interatomic decay to $\mathrm{Ar}_{2}^{3+}$. J. Chem. Phys., 128(1):014307, 2008.

[18] N. Saito, Y. Morishita, Suzuki I. H., S. D. Stoychev, A. I. Kuleff, L. S. Cederbaum, X.-J. Liu, H. Fukuzawa, G. Prümper, and K. Ueda. Evidence of radiative charge transfer in argon dimers. Chem. Phys. Lett., 441:16-19, 2007.

[19] K. Ueda, X.-J. Liu, G. Prümper, H. Fukuzawa, Y. Morishita, and N. Saito. Electron-ion coincidence momentum spectroscopy: Its application to Ar dimer interatomic decay. J. Electron. 
Spectrosc. Relat. Phenom., 155:113, 2007. experiment.

[20] S. Yan, P. Zhang, X. Ma, S. Xu, B. Li, X. L. Zhu, W. T. Feng, S. F. Zhang, D. M. Zhao, R. T. Zhang, D. L. Guo, and H. P. Liu. Observation of interatomic Coulombic decay and electron-transfer-mediated decay in high-energy electron-impact ionization of $\mathrm{Ar}_{2}$. Phys. Rev. A, 88(4), 2013.

[21] T. Pflüger, X. Ren, and A. Dorn. Electron-impact-induced dissociation of small argon clusters. Phys. Rev. A, 91(5), 2015.

[22] K. Kimura, H. Fukuzawa, T. Tachibana, Y. Ito, S. Mondal, M. Okunishi, M. Schöffler, J. Williams, Y. Jiang, Y. Tamenori, N. Saito, and K. Ueda. Controlling Low-Energy Electron Emission via Resonant-Auger-Induced Interatomic Coulombic Decay. J. Phys. Chem. Lett., 4:1838, 2013.

[23] P. O’Keeffe, E. Ripani, P. Bolognesi, M. Coreno, M. Devetta, C. Callegari, M. Di Fraia, K. C. Prince, R. Richter, M. Alagia, A. Kivimäki, and L. Avaldi. The Role of the Partner Atom and Resonant Excitation Energy in Interatomic Coulombic Decay in Rare Gas Dimers. J. Phys. Chem. Lett., 4:1797, 2013.

[24] P. Lablanquie, T. Aoto, Y. Hikosaka, Y. Morioka, F. Penent, and K. Ito. Appearance of interatomic Coulombic decay in Ar, Kr, and Xe homonuclear dimers. J. Chem. Phys., 127(15):154323, 2007.

[25] S. Scheit, L. S. Cederbaum, and H.-D. Meyer. Time-dependent interplay between electron emission and fragmentation in the interatomic Coulombic decay. J. Chem. Phys., 118:2092, 2003. theory.

[26] T. Ouchi, K. Sakai, H. Fukuzawa, I. Higuchi, Ph. V. Demekhin, Y.-C. Chiang, S. D. Stoychev, A. I. Kuleff, T. Mazza, M. Schöffler, K. Nagaya, M. Yao, Y. Tamenori, N. Saito, and K. Ueda. Interatomic Coulombic decay following Ne $1 s$ Auger decay in NeAr. Phys. Rev. A, 83(5), 2011.

[27] R. Dörner, V. Mergel, O. Jagutzki, L. Spielberger, J. Ullrich, R. Moshammer, and H. SchmidtBöcking. Cold Target Recoil Ion Momentum Spectroscopy: a 'momentum microscope' to view atomic collision dynamics. Phys. Rep., 330(2-3):95-192, 2000.

[28] J. Ullrich, R. Moshammer, A. Dorn, R. Dörner, L. Ph H. Schmidt, and H. Schmidt-Böcking. Recoil-ion and electron momentum spectroscopy: reaction-microscopes. Rep. Prog. Phys., 66(9):1463-1545, 2003. 
[29] T. Jahnke, Th. Weber, T. Osipov, A. L. Landers, O. Jagutzki, L.Ph.H. Schmidt, C. L. Cocke, M. H. Prior, H. Schmidt-Böcking, and R. Dörner. Multicoincidence studies of photo and Auger electrons from fixed-in-space molecules using the COLTRIMS technique. J. Electron. Spectrosc. Relat. Phenom., 141(2-3):229-238, 2004.

[30] E. A. Gislason. Series expansions for Franck-Condon factors. I. Linear potential and the reflection approximation. J. Chem. Phys., 58(9):3702, 1973.

[31] L. Ph H. Schmidt, T. Jahnke, A. Czasch, M. Schöffler, H. Schmidt-Böcking, and R. Dörner. Spatial Imaging of the $\mathrm{H}_{2}^{+}$Vibrational Wave Function at the Quantum Limit. Phys. Rev. Lett., 108(7):073202, 2012.

[32] B. Ulrich, A. Vredenborg, A. Malakzadeh, L. Ph. H. Schmidt, T. Havermeier, M. Meckel, K. Cole, M. Smolarski, Z. Chang, T. Jahnke, and R. Dörner. Imaging of the Structure of the Argon and Neon Dimer, Trimer, and Tetramer. J. Phys. Chem. A, 115(25):6936-6941, 2011.

[33] A. Kikas, S. J. Osborne, A. Ausmees, S. Svensson, O.-P. Sairanen, and S. Aksela. Highresolution study of the correlation satellites in photoelectron spectra of the rare gases. Journal of Electron Spectroscopy and Related Phenomena, 77(3):241-266, 1996.

[34] T. Miteva, Y.-C. Chiang, P. Kolorenč, A. I. Kuleff, K. Gokhberg, and Cederbaum L. S. Interatomic Coulombic decay following resonant core excitation of $\mathrm{Ar}$ in argon dimer. $J$. Chem. Phys., 141:064307, 2014.

[35] B. R. Brooks and H. F. Schaefer. The graphical unitary group approach to the electron correlation problem. Methods and preliminary applications. J. Chem. Phys., 70(11):50925106, 1979.

[36] B. R. Brooks, W. D. Laidig, P. Saxe, N. C. Handy, and H. F. Schaefer III. The Loop-Driven Graphical Unitary Group Approach: A Powerful Method for the Variational Description of Electron Correlation. Phys. Scr., 21(3-4):312, 1980.

[37] D. E. Woon and T. H. Dunning, Jr. Gaussian basis sets for use in correlated molecular calculations. III. The atoms aluminum through argon. J. Chem. Phys., 98:1358-1371, 1993.

[38] The diffuse $s$ and $d$, and the compact $d$ functions were generated as an even tempered sequence from the most diffuse $s$ and $d$, and the most compact $d$ basis functions with exponents $\xi=\alpha \beta^{l}$, where $\alpha$ is the exponent of the previous function, $\beta=10$ and $l=0.5$ for the compact exponent, $\beta=10$ and $l=-0.5,-1$ for the diffuse exponents. 
[39] Ph. V. Demekhin, Y.-C. Chiang, S. D. Stoychev, P. Kolorenč, S. Scheit, A. I. Kuleff, F. Tarantelli, and L. S. Cederbaum. Interatomic Coulombic decay and its dynamics in NeAr following K-LL Auger transition in the Ne atom. J. Chem. Phys., 131(10):104303, 2009.

[40] N. Sisourat, N. V. Kryzhevoi, P. Kolorenč, S. Scheit, T. Jahnke, and L. S. Cederbaum. Ultralong-range energy transfer by interatomic Coulombic decay in an extreme quantum system. Nat. Phys., 6:508, 2010.

[41] P. O'Keeffe, A. Ciavardini, E. Ripani, P. Bolognesi, M. Coreno, L. Avaldi, M. Devetta, M. Di Fraia, C. Callegari, K. C. Prince, and R. Richter. Experimental investigation of the interatomic coulombic decay in near dimers. Physical Review A, 90(4), 2014.

[42] V. Averbukh and L. S. Cederbaum. Ab initio calculation of interatomic decay rates by a combination of the Fano ansatz, Green's-function methods, and the Stieltjes imaging technique. J. Chem. Phys., 123:204107, 2005.

[43] F. Mertins and J. Schirmer. Algebraic propagator approaches and intermediate-state representations. I. The biorthogonal and unitary coupled-cluster methods. Phys. Rev. A, 53(4):2140$2152,1996$.

[44] A. Nicklass, M. Dolg, H. Stoll, and H. Preuss. Ab initio energy-adjusted pseudopotentials for the noble gases Ne through Xe: Calculation of atomic dipole and quadrupole polarizabilities. J. Chem. Phys., 102(22):8942-8952, 1995.

[45] K. Kaufmann, W. Baumeister, and M. Jungen. Universal Gaussian basis sets for an optimum representation of Rydberg and continuum wavefunctions. J. Phys. B: At. Mol. Opt. Phys., 22(14):2223, 1989.

[46] Ying-Chih Chiang, Frank Otto, Hans-Dieter Meyer, and Lorenz S. Cederbaum. Kinetic energy release in fragmentation processes following electron emission: a time-dependent approach. The Journal of chemical physics, 136(11):114111, 2012.

[47] E. Pahl, H.-D. Meyer, and L. S. Cederbaum. Competition between excitation and electronic decay of short-lived molecular states. Zeitschrift fr Physik D Atoms, Molecules and Clusters, 38(3):215-232, 1996. 